\title{
Root Canal Medicaments - Literature Review
}

\author{
Salma A Salama ${ }^{1}$ and Aya A Salama ${ }^{2 *}$ \\ ${ }^{1}$ Faculty of Dentistry (Medical Sciences-Biochemistry Department)-British University \\ of Egypt \\ ${ }^{2}$ Lecturer, Department of Fixed Prosthodontics, Faculty of Dentistry, MSA University, \\ Egypt
}

*Corresponding Author: Aya Salama, Lecturer, Department of Fixed Prosthodontics, Faculty of Dentistry, MSA University, Egypt. E-mail: aassalama@hotmail.com

DOI: 10.31080/ASMS.2020.04.0569
Received: January 18, 2020

Published: February 19, 2020

(C) All rights are reserved by Salma A Salama and Aya A Salama.

\begin{abstract}
Objective: Identify, through an integrative literature review, the general update on clinical studies, current research and new developments in the area of root canal pastes, endodontic cements and dressings.

Method: The scientific electronic bases were used: MEDLINE, PUBMED, and COCHRANE for assessing ADM's class III medical devices: Odontopaste, Odontocem and Odontocide, to determine if these products are in line with recent best dental practice.The research took place between March and December 2019, for articles published between the period 2007 to 2019 . Exclusion criteria were: editorials, abstracts, theses and dissertations.

Results: 21 articles were found that reflected relevant aspects of the medical root canal medicaments.

Conclusion: For a wide range of materials currently available on the market, ADM's class III medical devices: Odontopaste, Odontocem and Odontocide meet the requirements of a formulation which is compatible with recent restorative and endodontic practice and in regards the active ingredients in all three products no safety complains was reported from any in the last 12 months research.
\end{abstract}

Keywords: Root Canal Therapy; Endodontics; And Root Canal Obturation

\section{Introduction}

The mechanical preparation of a root canal is not sufficient to completely eliminate bacteria from the root canal. The use of an intra-canal medicament placed in-between appointments has been suggested to achieve maximum reduction of bacterial load prior to obturation [1]. Medicaments inside the antimicrobial agents must be efficient enough to pass and penetrate the dentinal tubules, apical root canal deltas and accessory canals and at the same time preserving their concentration that will eliminate disease causing bacteria [2].

\section{Objective}

The aim of this review article is to provide a general update on clinical studies, current research and new developments in the area of root canal pastes, endodontic cements and dressings which have gained specific attention in last 12 months. This is along with assessing medical root canal treatments Odontopaste, Odontocem and Odontocide, to determine if these products are in line with recent best dental practice.

\section{Review of Literature}

\section{Odontopaste}

In a recent review (Ramaprabha B. and Srilekha J. 2017) [3] discussed antibiotics use in Endodontics. Odontopaste was described as a zinc-oxide based root canal paste with $5 \%$ clindamycin hydrochloride which is a bacteriostatic, broad spectrum antibiotic and $1 \%$ triamcinolone acetonide, anti-inflammatory steroidal agent provides pain relief (ADM, Brisbane, Queensland, Australia) [4].

In a systematic review research by Esra., et al. 2017 [1], a case study was included describing the treatment done for two teeth in same patient, where one tooth was treated by apexification and 
endodontic treatment with mineral trioxide aggregate, and the second one was treated with regenerative endodontics. The two teeth developed periapical healing with good evidence of root growth, root walls thickening and complete apex formation. Regarding revascularization, there was a positive response to pulp testing and this was attributed to disinfection protocol used by utilizing of Odontopaste. They presumed that triamcinolone (corticosteroid based) included in Odontopaste may have encouraged the differentiation of odontoblast like cells and dentinogenesis. They also stated that corticosteroids are other substitutes to traditional disinfection methodologies; however they need to be investigated by future research considering drawbacks, as well.

A study was performed by Jarrett., et al. [5], aimed to develop techniques that quantify main components of dental medicament pastes for root canal treatment, and to evaluate if change in composition occurred during storage. As it is possible that degradation might occur during storage, through events such as precipitation, phase separation, or oxidation, this will impair its therapeutic properties and usefulness in the clinical setting. Regarding Odontopaste intra-canal medicament, the paste composition was Clindamycin, Triamcinolone, Calcium hydroxide, Polyethylene glycol, zinc oxide and Calcium chloride. The reported composition of Clindamycin $\%$ by the manufacture was $5 \%$, the measured content in this study in new paste was $5.57 \%$ with 0.65 Standard deviation) and for an expired paste $4.13 \%$ with (1.13) Standard deviation). The reported composition of Triamcinolone by the manufacture was $1 \%$, the measured content in this study in new paste was $0.55 \%$ with ( 0.06 Standard deviation) and for an expired paste $0.49 \%$ with (0.11) Standard deviation). In terms of the validity of label concentrations of materials, the present results revealed that the calculated levels of extracted core active ingredients of the medicament pastes tested (Dental paste, Odontopaste, Doxypaste and Ledermix) were marginally lower than the manufacturers' reported compositions. This refined targeted Mass Spectrometric analytical technique will hopefully allow for accurate assessment of diffused primary endodontic medicament through root dentine, thus allowing the determination of an effective drug dose.

Regarding Odontopaste efficacy in pulpal necrosis and infection, a case study was discussed in brief [6]. A 7 year and 10 month old girl fall off her bicycle. She developed a throbbing pain, extraoral swelling and rapidly increased in size in tooth 11. It was found to be associated with an apical infection, acutely tender to palpa- tion and showed increased mobility. She was diagnosed with acute periapical periodontitis with an associated facial cellulitis. Pulpal necrosis and infection in an immature tooth with large pulp chambers, thin dentine root walls and wide dentinal tubules is a challenging situation [7]. After 24 hour of intravenous amoxicillin, the root canal space was irrigated with copious amounts of sodium hypochlorite (1\%) and dressed with Odontopaste. On review 1 month later, tooth was not tender to percussion and mobility was within normal limits [6].

A study by Plutzer., et al. 2017 [8], compared the efficacy of commonly used medicaments against Enterococcus faecalis. Each test medicament (Ledermix, $\mathrm{Ca}(\mathrm{OH}) 2$, Odontoposte, $0.2 \%$ chlorohexidine and 50:50 combinations of Odontopaste/ $\mathrm{Ca}(\mathrm{OH}) 2)$. Sodium hypochlorite achieved total bacterial elimination. Ledermix paste and Odontopaste eliminated bacteria by $35.5 \%$ and $71.1 \%$, respectively which suggests that Ledermix failed to eliminate E. faecalis from the root canal. A statistical significant difference $(p<0.05)$ was found between Ledermix and Odontopaste which suggests that Odontopaste is superior to Ledermix. $\mathrm{Ca}(\mathrm{OH}) 2 / 0$ dontopaste reduced the viability by $>99 \%$, which is highly effective in reducing bacterial viability. This is contradictory with what (Ramaprabha B. and Srilekha J. 2017) in their review, they elaborated that no real advantages of mixing calcium hydroxide with odontopsate, as there was no significant improvement in antibacterial action when mixing with calcium hydroxide, added to it its inhibition to steroid activity of triamcinolone acetonide [4].

A major reason for the lack of effectiveness of either Ledermix paste or Odontopaste is the intrinsic resistance of E. faecalis to several commonly used antibiotics and perhaps more importantly, its ability to acquire resistance to all currently available antibiotics, either by mutation or by horizontal gene transfer [9].

Segura-Egea., et al. in 2017, [10], reviewed the current literature of antibiotics, make recommendations for their prescription in endodontic patients regarding their indication and safety. They mentioned that risk of adverse effects following systemic application has led to the use of locally applied antibiotics in root canal treatment. Most Regenerative endodontic procedures include minimal to no mechanical debridement (Diogenes., et al. 2013) [11], relying on chemical debridement and on the use of intracanal medicaments to achieve disinfection. Therefore, intracanal medicaments have been used in almost all published case reports [12]. 
The review also stated that there is no scientific evidence to support the use of antibiotics in pulp capping procedures [13].

In a recent review in August 2017 [14], Safety of Clindamycin was questioned. Clindamycin is an excellent antibiotic for odontogenic infections; its mechanism of action and spectrum of activity, make it particularly advantageous. It is an integral component of many antibiotic prophylaxis and treatment guidelines, as supported by international scientific authorities such as the World Health Organization, American Heart Association, and the American Dental Association. The concern that clindamycin is a "dangerous" drug, is highly associated with the development of Clostridium infection, which is the result from unfortunate cases with intravenous doses administered. But, when being an integral proportion of intra-oral medicaments with the appropriate dosage, clindamycin should continue to be considered as one of the most effective and safe medicines.

\section{Odontocem}

Calcium silicate cements are by so far, the most common and used materials in endodontic treatment. Odontocem is a calcium silicate based technology added to it $0.2 \%$ low steroid Triamcinolone acetonide, with excellent handling characteristics and biocompatibility. It can be used in dentine substitution, endodontic therapy and liner. Their proven biocompatibility and ability to initiate calcium and phosphate incorporation at the interface with the periodontal tissue have a primary role in bone tissue repair [15].

A Systematic Review and Meta-Analyses statement was done in 2017 by Możyńska J., et al [16]. The literature search was based on all publications with a year limit, on the effect of different calcium silicate-based cements on dental tissue discoloration. The results indicated that some materials showed a strong potential for staining, including MTA Angelus, ProRoot MTA, and Ortho MTA. While Biodentine, Retro MTA, Portland cement, Endo Sequence Root Repair Material, Odontocem (Australian Dental Manufacturing, Brisbane, Australia), MM-MTA, and MTA Ledermix were materials with the smallest staining potential, which was nearly invisible to the human eye $(\Delta \mathrm{E}<3.3)$.

It was concluded by Teoh Yu., et al. 2017 [17] that no root filling material was completely able to prevent bacterial penetration when faced with a constant challenge from salivary microorganisms. Within the timeframe and limits of this study, hard-setting alkaline cements show greater resistance to bacterial penetration.

\section{Odontocide}

Calcium hydroxide is mostly used for its antiseptic properties. Regarding its role against endodontic biofilms, studies showed that calcium hydroxide is not as effective as other substances used as intracanal medications. Calcium hydroxide demonstrates a very low efficiency against microorganisms and should be used for its other biological and chemical properties not as antimicrobial [18].

Calcium hydroxide endodontic pastes water based, limit the dissolution of calcium hydroxide. Using non-aqueous solvents such as the polyethylene glycols (PEG 400) which is colorless, and hygroscopic polymer, a greater dissolution and faster hydroxyl ion release can be achieved, leading to enhanced antimicrobial actions, and improvements in performance and biocompatibility. Products based on PEG 400 show greater measured alkalinity despite having a much lower total calcium hydroxide content [19]. When inspecting from a clinical point of view, calcium hydroxide dissolution allows the medical component to work over a long period of time and decrease the necessity of redressing the root canals, especially when there is a chronic peri-apical infection [20].

Saturated aqueous solutions of calcium hydroxide are much more subjected to rapid inactivation by dentine buffering than medicament pastes with viscous based vehicles. When PEG-based medicaments (such as Odontocide) are assessed, up to $140 \mathrm{~min}$ is required before buffering is reached. A study ranked medicaments from most alkaline to least alkaline where Calmix was highest, followed by Odontocide, Pulpdent and then Calasept Plus [19]. Odontocide is $20 \%$ calcium hydroxide (PEG 400 and water based) with alkaline $\mathrm{Ph}$ of 13.2 [21]. The inclusion of ibuprofen significantly reduce the incidence of postoperative pain as compared to medicaments with non-steroidal anti-inflammatory component [22].

In 2017, Zilm P., et al. [23] studied the effectiveness of D-amino acids (DAAs) in reducing biofilm growth in the presence of subminimal concentrations of sodium hypochlorite and Odontocide $(0.25 \% \mathrm{w} / \mathrm{v})$, and in the presence of Odontopaste $(0.25 \% \mathrm{w} / \mathrm{v})$. When only the endodontic agent was included, the biofilm grown over 1 day was significantly reduced in the presence of sub-MIC of Odontocide, $\mathrm{NaOCl}$ and Odontopast. Only $\mathrm{NaOCl}$ had the effect of reducing the biofilm level when left for 3 days and none of the agents significantly reduced biofilm growth over 6 days. When Damino acids (DAAs) where added to Odontocide and Odontopaste, there were significantly decrease in biofilm growth at 1, 3 and 6 days; compared to (Odontocide or Odontopaste only). 
The research hypothesized that dentinal tubules buffer the $\mathrm{pH}$ of the calcium hydroxide present in medicaments such as Odontocide by absorbing hydroxyl ions which creates an environment containing sub-lethal levels of medicament that can potentially promote biofilm growth, and the presence of DAAs in the medicament may allow diffusion of the DAAs down the dentinal tubules to reduce biofilms and render organisms more susceptible to killing [23].

\section{Conclusion}

For a wide range of materials currently available on the market, ADM's class III medical devices: Odontopaste, Odontocem and Odontocide meet the requirements of a formulation which is compatible with recent restorative and endodontic practice and in regards the active ingredients in all three products no safety complains was reported from any in the last 12 months research.

\section{Conflict of Interest}

The authors declare no conflict of interest.

\section{Bibliography}

1. Esra P., et al. "Revascularization: a review of clinical reports on a contemporary treatment modality for endodontics". Biomedical Research 28.2 (2017): 644-656.

2. Wilson C., et al. "Clonal diversity in biofilm formation by Enterococcus faecalis in response to environmental stress associated with endodontic irrigants and medicaments". International Endodontic Journal 48 (2015): 210-219.

3. Ramaprabha B and Srilekha J. "Antibiotics in endodontics - A concise review". International Journal of Applied Dental Sciences 3.4 (2017): 323-329.

4. Athanassiadis M., et al. "The effect of calcium hydroxide on the steroid component of ledermix and odontopaste". International Endodontic Journal 44.12 (2011): 1161-1169.

5. Jarrett J., et al. "Development of techniques for determination of primary components of dental medicament paste mixtures for root canal treatment". Journal of Investigative and Clinical Dentistry (2017): e12294.

6. D'Mello G and Moloney L. "Management of coronal discolouration following a regenerative endodontic procedure in a maxillary incisor". Australian Dental Journal 62 (2017): 111-116.

7. Lenherr P., et al. "Tooth discoloration induced by endodontic materials: a laboratory study". International Endodontic Journal 45 (2012): 942-949.
8. Plutzer B., et al. "Comparative efficacy of endodontic medicaments and sodium hypochlorite against Enterococcus faecalis biofilms". Australian Dental Journal (2017).

9. Sood S., et al. "Enterococcal infections and antimicrobial resistance". Indian Journal of Medical Research 128.2 (2008):111121.

10. Segura-Egea J., et al. "Antibiotics in Endodontics: a review". International Endodontic Journal 50 (2017): 1169-1184.

11. Diogenes A., et al. "An update on clinical regenerative endodontics". Endodontic Topics 28 (2013): 22-23.

12. Kontakiotis EG., et al. "Regenerative endodontic therapy: a data analysis of clinical protocols". Journal of Endodontics 41(2015): 146-154.

13. Li Z., et al. "Direct pulp capping with calcium hydroxide or mineral trioxide aggregate: a meta-analysis". Journal of Endodontics 41 (2015): 1412-1417.

14. Donaldson M and Goodchild JH. "Is clindamycin dangerous?" General Dentistry 65.4 (2017):12-15.

15. Sonja A., et al. "Biodentine as a furcal perforation repair material - A case series”. Medicinski Pregled 7.8 (2017): 223-225.

16. Joana M., et al. "Tooth Discoloration Induced by Different Calcium Silicate-based Cements: A Systematic Review of In-Vitro Studies". American Association of Endodontists 43.10 (2017): 1593-1601.

17. Teoh Yu., et al. "Sealing Ability of Alkaline Endodontic Cements versus Resin Cements”. Materials 10.11 (2017): 1-7.

18. Andrada T., et al. "Antibacterial and antifungal activity of endodontic intracanal medications". Clujul Medical 90.3 (2017): 344-347.

19. Athanassiadis B and Walsh L. "Aspects of Solvent Chemistry for Calcium Hydroxide Medicaments". Materials 10 (2017): 1219.

20. Madarati AA., et al. "Preference and usage of intracanal medications during endodontic treatment". Saudi Medical Journal 38 (2017): 755-763.

21. Teoh YY., et al. "The influence of aqueous and PEG 400 solvent vehicles on hydroxyl ion release from calcium hydroxide medicaments". International Dental Journal 11 (2016): 42-50.

22. Australian Society of Endodontology (WA) (2017). 
23. Zilm P., et al. "D-amino acids reduce Enterococcus faecalis biofilms in vitro and in the presence of antimicrobials used for root canal treatment". Plos ONE 12.2 (2017): e0170670.

\section{Assets from publication with us}

- Prompt Acknowledgement after receiving the article

- Thorough Double blinded peer review

- Rapid Publication

- Issue of Publication Certificate

- High visibility of your Published work

Website: https://www.actascientific.com/

Submit Article: https://www.actascientific.com/submission.php Email us: editor@actascientific.com

Contact us: +919182824667 